Communications in Physics, Vol. 24, No.3S1 (2014), pp. 40-44

DOI:10.15625/0868-3166/24/3S1/5075

\title{
APPLICATION OF PATH-INTEGRAL FOR STUDYING EXAFS CUMULANTS
}

\author{
HO KHAC HIEU \\ Duy Tan University, Da Nang, Viet Nam \\ NGUYEN MANH HAI \\ Hanoi University of Science, Vietnam National University, Hanoi \\ E-mail: hieuhk@duytan.edu.vn
}

Received 04 April 2014

Accepted for publication 24 May 2014

\begin{abstract}
In this work, the path-integral effective potential (PIEP) method has been applied to re-study the temperature dependence of extended X-ray absorption fine structure (EXAFS) cumulants of materials. Using the trial density matrix and effective potential expression, we derived the analytical expressions of the first three EXAFS cumulants in the first shell of materials. The cumulant relation is also calculated to determine the temperature range in which the PIEP method could be applied. Our results are compared with available experimental data as well as with those calculated by the first-order perturbation approach in anharmonic Einstein model and the reasonable agreements are achieved.
\end{abstract}

Keywords: EXAFS cumulants, path-integral, effective potential.

\section{INTRODUCTION}

On the determination of the local structures of materials, the extended X-ray Absorption Fine Structure (EXAFS) spectroscopy is one of powerful techniques. The EXAFS data can be theoretically analyzed by means of the cumulant expansion method [1]. There are several approaches used to study the temperature dependence of anharmonic EXAFS cumulants such as perturbation approach in Einstein model [2] and Debye model [3], and statistical moment method [4]. However, most of these methods still can not be valid at very high temperature due to the strong anharmonic contributions. Recently, the path-integral effective potential (PIEP) method has been efficiently used to theoretically study thermodynamic properties of materials [5-8]. This method has included the anharmonic contributions as well as the quantum effects.

In present paper, in order to expose the efficiency of PIEP on studying the thermodynamic properties of materials, we are going to re-investigate the EXAFS cumulants of diatomic $\mathrm{Br}_{2}$ by PIEP in the wide range of temperature from 0 to $800 \mathrm{~K}$. Our calculations for the first three EXAFS cumulants are going to be compared with the results of the first-order perturbation theory (PT) and experimental data when possible.

(C)2014 Vietnam Academy of Science and Technology 


\section{FORMALISM}

In this section, we firstly briefly report the results of the PIEP method that have been established by Cuccoli [5], Yokoyama [6,7] and Miyanaga et. al. [8]. In the quantum statistical physics, the average of a physical quantity $\langle A\rangle$ can be calculated by

$$
\langle A\rangle=\frac{1}{Z} \int d x \rho(x) A(x)
$$

where $\rho(x)$ is the density matrix and $\mathrm{Z}$ is the partition function.

In the PIEP theory, the trial Euclidean action $A_{0}[x(u)]$ is assumed as the harmonic action with the trial density matrix for two-body case has the form as

$$
\rho_{0}(\bar{x}) \cong \sqrt{\frac{\mu}{2 \pi \hbar^{2} \beta}} e^{-\beta V_{e f f}(\bar{x})} \frac{1}{\sqrt{2 \pi \alpha(\bar{x})}} \int_{-\infty}^{\infty} d x e^{-\frac{(x-\bar{x})^{2}}{2 \alpha(\bar{x})}}
$$

where $\beta=1 / k_{B} T, k_{B}$ is the Boltzmann constant, $\bar{x}=\frac{1}{\beta \hbar} \int_{0}^{\beta \hbar} d u x(u)$ is the average path; $\alpha(\bar{x})$, $A_{0}[x(u)]$ and $V_{\text {eff }}(\bar{x})$ are, respectively, the pure quantum fluctuation, trial Euclidean action and effective potential, which are correspondingly defined as follows

$$
\begin{gathered}
\alpha(\bar{x})=\frac{\hbar}{2 \mu \omega(\bar{x})}\left(\operatorname{coth} f(\bar{x})-\frac{1}{f(\bar{x})}\right), f(\bar{x})=\frac{\beta \hbar \omega(\bar{x})}{2} \\
A_{0}[x(u)]=\int_{0}^{\beta \hbar} d u\left[\frac{1}{2} \mu \dot{x}^{2}+\frac{1}{2} \mu \omega^{2}(x) x^{2}+w(\bar{x})\right] \\
V_{\text {eff }}(\bar{x})=w(\bar{x})+\frac{1}{\beta} \ln \frac{\sinh f(\bar{x})}{f(\bar{x})}
\end{gathered}
$$

The variational parameters $w(\bar{x})$ and $\omega^{2}(x)$ can be optimized by using the Jensen-Feynman inequality $[6,7]$ as

$$
F \leqslant F_{0}+\frac{1}{\beta \hbar}\left\langle A-A_{0}\right\rangle_{0}
$$

where $F$ and $F_{0}$ are the true and trial free energies of system.

The expressions of $w(\bar{x})$ and $\omega^{2}(x)$ have been reported in [8] as

$$
\begin{gathered}
\omega^{2}=\omega_{0}^{2}+12 \frac{k_{4}}{\mu} \alpha-6 \frac{k_{3}}{\mu} \bar{x}+12 \frac{k_{4}}{\mu} \bar{x}^{2} \\
w=\frac{k_{0}}{2} \bar{x}^{2}-k_{3} \bar{x}^{3}+k_{4}\left(\bar{x}^{4}-3 \alpha^{2}\right)
\end{gathered}
$$

here, $k_{0}, k_{3}$ and $k_{4}$ are the harmonic, third-order and fourth-order force constants, respectively, which describe the interatomic potential $V(r)$ between two-body system as

$$
V(r) \approx \frac{1}{2} k_{0}\left(r-r_{0}\right)^{2}-k_{3}\left(r-r_{0}\right)^{3}+k_{4}\left(r-r_{0}\right)^{4}
$$


Thus, the first three EXAFS cumulants of materials within the effective-potential approximation now can be determined as follows

$$
\begin{aligned}
\sigma^{(1)} & =\left\langle r-r_{0}\right\rangle \cong \frac{1}{Z_{0}} \int \bar{x} \rho_{0}(\bar{x}) d \bar{x} \\
\sigma^{2} & =\sigma^{(2)}=\left\langle\left(r-r_{0}-\sigma^{(1)}\right)^{2}\right\rangle \cong \frac{1}{Z_{0}} \int \bar{x}^{2} \rho_{0}(\bar{x}) d \bar{x}-\left[\sigma^{(1)}\right]^{2} \\
\sigma^{(3)} & =\left\langle\left(r-r_{0}-\sigma^{(1)}\right)^{3}\right\rangle \cong \frac{1}{Z_{0}} \int \bar{x}^{3} \rho_{0}(\bar{x}) d \bar{x}-3 \sigma^{(1)} \sigma^{2}-\left[\sigma^{(1)}\right]^{3}
\end{aligned}
$$

where $Z_{0}$ is the trial partition function.

Using these above results, we can calculate the first three EXAFS cumulants of $\mathrm{Br}_{2}$ numerically.

\section{NUMERICAL CALCULATIONS AND DISCUSSIONS}

In this section, the derived results are applied to numerically calculate the EXAFS cumulants of diatomic $\mathrm{Br}_{2}$. The force constants of $\mathrm{Br}_{2}$ have been proposed as in [6,9]: the harmonic force constant $k_{0}=2.459 .10^{-8}(\mathrm{~N} / \AA)$, the third and fourth order force constants $k_{3}=1.756 .10^{-8}$ $\left(\mathrm{N} / \AA^{2}\right)$ and $k_{4}=1.058 .10^{-8}\left(\mathrm{~N} / \AA^{3}\right)$.

In the Figs. 1-3, we present the PIEP as well as first-order PT calculations for the first three EXAFS cumulants of diatomic $\mathrm{Br}_{2}$ as the functions of temperature $T$. As it can be seen from the Figs. $2 \& 3$, the PIEP results are very good in agreement with the available experimental EXAFS data [10] and in Figs. $1 \& 2$, at low temperature $T \leq 300 \mathrm{~K}$ the PIEP results are in consistent with those of PT calculations. At low temperature, these results contain the values $\sigma_{0}^{(1)}=0.280 .10^{-2}(\AA)$ and $\sigma_{0}^{(2)}=0.131 .10^{-2}\left(\AA^{2}\right)$ which correspond to the zero-point vibrations of the first and second order EXAFS cumulants of diatomic $\mathrm{Br}_{2}$. It denotes that the quantum effects have been included in these evaluations. At temperature $T>300 \mathrm{~K}$, the present PIEP determinations are greater than those of PT calculations. It is because of the increasing of anharmonicity when temperature increases. In the first order PT with Eintein model, it assumes that all atoms vibrate with the same frequency $\omega_{E}$ for all temperature range which corresponds to the neglection of the anharmonicity at high temperature. Moreover, by fitting method, we confirm that the $\sigma^{(1)}$ and $\sigma^{(2)}$ in PT calculations are proportion to $T$ at high temperature (about $T>300 \mathrm{~K}$ ) while the results of PIEP are not really linear to $T$ (seemly proportion to $T^{2}$ ). Our fitting parameters at $T>400 \mathrm{~K}$ of PIEP and PT approaches are shown in Table 1.

We show the third EXAFS cumulant of $\mathrm{Br}_{2}$ in Fig. 3. At the temperature $T>100 \mathrm{~K}$, the similar trends are still observed as in Figs. 1\&2. However, there is a strange behavior of PIEP $\sigma^{(3)}$ at $T_{i} 100 \mathrm{~K}$ : It decreases little by little to zero at $T=0 \mathrm{~K}$ limit which means that in this case, the zero-point vibration (or quantum fluctuation) has been neglected. There are some reasons which can simply explain this difference: (1) the one-dimensional calculation for diatomic $\mathrm{Br}_{2}$; (2) the vibrational properties tends to be harmonic at $0 \mathrm{~K}$ [7]. This result implies that, the PT method should be used instead of PIEP in evaluating EXAFS $\sigma^{(3)}$ of $\mathrm{Br}_{2}$ at low temperature. In order to cross-check this conclusion, we make a calculation of the cumulant relation $\sigma^{(1)} \sigma^{2} / \sigma^{(3)}$ which can be considered as the cumulant investigation standard for $\mathrm{Br}_{2}$ in the temperature range $0-$ $800 \mathrm{~K}$. Results of PIEP as well as first-order PT approach are showed in the Fig. 4. The values 


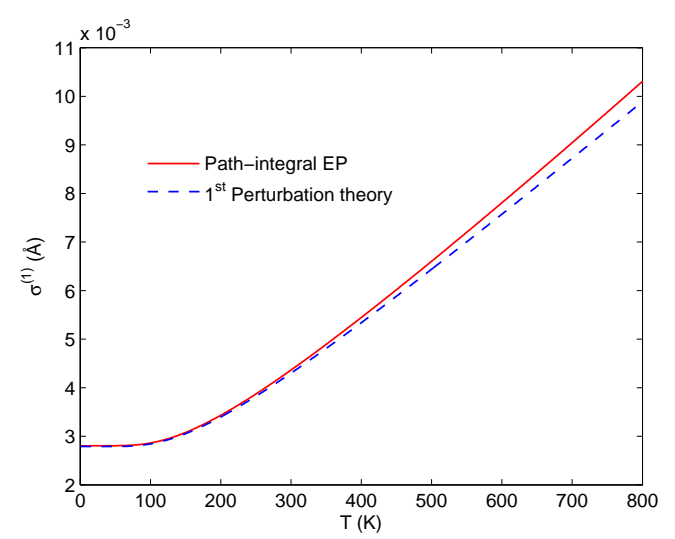

Fig. 1. Temperature-dependence of the first EXAFS cumulant of $\mathrm{Br}_{2}$.

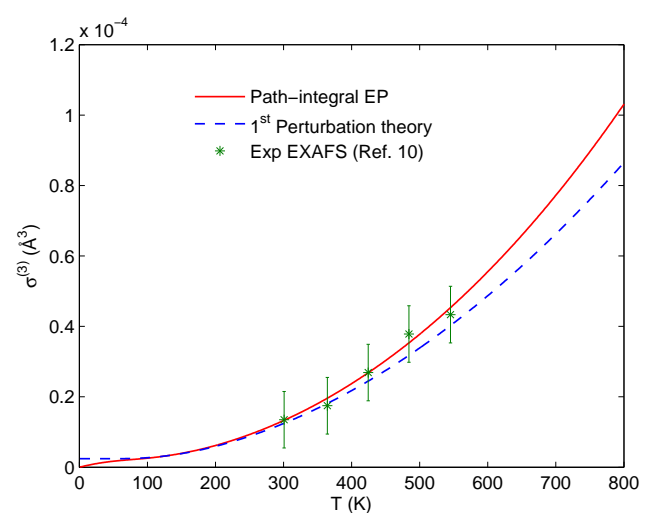

Fig. 3. Temperature-dependence of the third EXAFS cumulant of $\mathrm{Br}_{2}$.

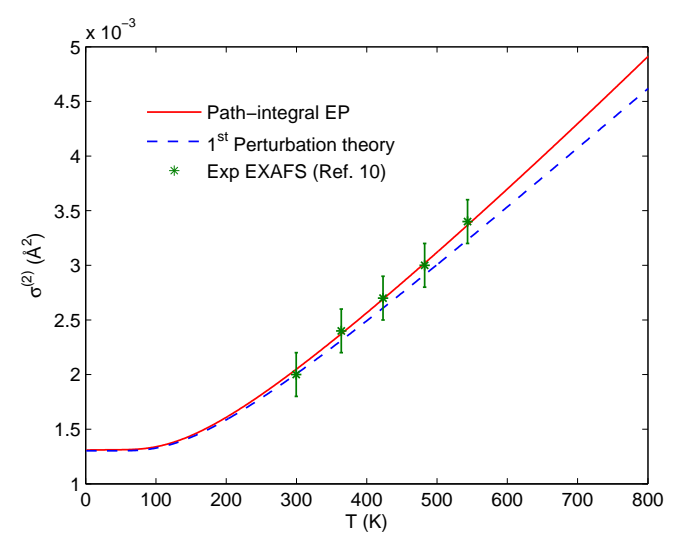

Fig. 2. Temperature-dependence of the second EXAFS cumulant of $\mathrm{Br}_{2}$.



Fig. 4. Temperature-dependence of the cumulant relation $\sigma^{(1)} \sigma^{2} / \sigma^{(3)}$ of $\mathrm{Br}_{2}$.

of cumulant relation of these two methods little by little reduce to the constant value of $1 / 2$ at temperature about $700 \mathrm{~K}$. Furthermore, at temperature below $100 \mathrm{~K}$, the cumulant relation in PIEP model reach very-high value which does not satisfy the condition obtained from experiment [11] before. This result denotes that PIEP method is not suitable for evaluating the third order EXAFS cumulant at low temperature. At temperature $T>100 \mathrm{~K}$, the third EXAFS cumulant $\sigma^{(3)}$ calculated by PIEP as well as PT trends to proportion to the square of temperature $T^{2}$. We display the fitting parameters of PIEP and PT results in Table 1.

\section{CONCLUSIONS}

In this work, the PIEP approach has been applied to study the temperature dependence of EXAFS cumulants of diatomic materials. Using this trial density matrix expression, we have performed numerical calculations of first three EXAFS cumulants for diatomic $\mathrm{Br}_{2}$. Our results are in agreement with available experimental data as well as with those calculated by the firstorder perturbation approach. This research has pointed out the potential of PIEP method on study 
Table 1. Results of fitting PIEP and PT calculations (in temperature range $T>400 \mathrm{~K}$ ) as functions $\sigma^{(n)}=a_{0}+a_{1} T+a_{2} T^{2}, n=1,2,3$.

\begin{tabular}{ccccccc}
\hline \hline & $\sigma^{(1)}(P T)$ & $\sigma^{(2)}(P T)$ & $\sigma^{(3)}(P T)$ & $\sigma^{(1)}($ PIEP $)$ & $\sigma^{(2)}($ PIEP $)$ & $\sigma^{(3)}($ PIEP $)$ \\
\hline \hline$a_{0}$ & $7.41 .10^{-4}$ & $3.46 .10^{-4}$ & $4.61 .10^{-7}$ & $1.11 .10^{-3}$ & $5.28 .10^{-4}$ & $7.91 .10^{-6}$ \\
$a_{1}$ & $1.14 .10^{-5}$ & $5.33 .10^{-6}$ & $-1.04 .10^{-9}$ & $1.01 .10^{-5}$ & $4.69 .10^{-6}$ & $-3.90 .10^{-8}$ \\
$a_{2}$ & 0 & 0 & $1.36 .10^{-10}$ & $1.70 .10^{-9}$ & $9.87 .10^{-10}$ & $1.97 .10^{-10}$ \\
\hline \hline
\end{tabular}

thermodynamic and mechanical properties of materials such as thermal expansion, bulk modulus, mean-square fluctuation...

\section{ACKNOWLEDGMENTS}

The authors would like to gratefully thank Prof. Toshihiko Yokoyama and Prof. Nguyen Suan Han for useful comments. This research is funded by Vietnam National Foundation for Science and Technology Development (NAFOSTED) under grant number 103.02-2012.06.

\section{REFERENCES}

[1] E. D. Crozier, J. J. Rehr, and R. Ingalls, X-ray Absorption, edited by D. C. Koningsberger and R. Prins (Wiley, New York, 1988).

[2] A. I. Frenkel and J. J. Rehr, Phys. Rev. B 48 (1993) 585.

[3] N. V. Hung, N. B. Trung, and B. Kirchner, Physica B 405 (2010) 2519.

[4] V. V. Hung, H. K. Hieu and K. Masuda-Jindo, Comput. Mater. Sci. 49 (2010) S214.

[5] A. Cuccoli, R. Giachetti, V. Tognetti, R. Vaia and P. Verrucchi, J. Phys. Condens. Matter 7 (1995) 7891.

[6] T. Yokoyama, Phys. Rev. B 57 (1998) 3423.

[7] T. Yokoyama, J. Synchrotron Rad. 6 (1999) 323.

[8] T. Miyanaga, T. Suzuki and T. Fujikawa, J. Synchrotron Rad. 7 (2000) 95.

[9] K. P. Huber and G. Herzberg, Molecular Spectra and Molecular Structure IV: Constants of Diatomic Molecules (Van Nostrand Reinhold, New York, 1979).

[10] T. Yokoyama, K. Kobayashi, T. Ohta, A. Ugawa, Phys. Rev. B 53 (1996) 6111.

[11] E.A. Stern, P. Livins, Z. Zhang, Phys. Rev. B 43 (1991) 8850. 